\title{
Enterovirus Meningitis in Children from Constanta
}

${ }^{1}$ Clinical Infectious Diseases Hospital, Constanta, Romania

${ }^{2}$ Faculty of Medicine, University "Ovidius" of Constanta, Romania

\begin{abstract}
Introduction: Many different viruses can cause meningitis. Most children are exposed to some of them during their life without developing meningitis. Of all the viruses, enteroviruses are the most frequent involved in etiology of meningitis.

Material and Method: The evaluated group consisted of 73 patients, boys and girls aged 1 to 12 years, hospitalized at Constanta Clinical Infectious Diseases Hospital during the period May 2016 - October 2017.

Results: Over the studied period, 243 patients were admitted with suspicion of meningitis, but just 73 of them were confirmed $(30.04 \%)$. The average age of children with Viral Meningitis was 5 years and 8 months old, with a gender distribution that revealed a 2:1 balance in boy's favor. The most affected age groups were 1-3 years and 4-6 years with 27 cases each. Fever, headaches and vomiting were the most common symptoms, occurring in majority of the patienst (53/73). Cerebrospinal fluid was clear in 58 cases, meanwhile in 15 cases the fluid was opalescent. Polymerase chain reaction on cerebrospinal fluid revealed Enterovirus in 27 out of the 73 cases.

Conclusions: Viral meningitis with enteroviruses should be considered in any paediatric patients admitted with fever, headaches and vomiting, boys and girls, due to the potential outbreaks that can appear during the summer or autumn.
\end{abstract}

Keywords: children, viral meningitis, enterovirus, outbreak

Simona Claudia Cambrea

Faculty of Medicine, "Ovidius” University, Constanta, Romania

Ferdinand Avenue 100, Constanta, Romania

email : cambrea.claudia@gmail.com

\section{Introduction}

Meningitis is an important source of morbidity and mortality. The most common symptoms include sudden high fever, headache, vomiting without nausea, confusion, neck stiffness, and photophobia [1]. Viral meningitis is more frequent than bacterial meningitis and is generally less severe $[1,2]$.

The most common clinical challenge for physicians is whether a meningitis case has a viral or bacterial etiology, as this will influence the therapeutic attitude [3]. The most common viral meningitis are those involving enteroviruses [1]. From the enteroviruses, Coxsakie and Echoviruses are the most common groups involved in etiology of meningitis $[2,4,5]$.

In Romania, a country with temperate climates, enterovirus infections are commonest during the summer and autumn, and spread is predominantly through the fecal-oral route, with infections often commonly acquired in kindergarten"[2]. Children with viral meningitis usually recover completely just with symptomatic and pathogenic treatment $[1,2]$.

\section{Objective}

We focused our attention on an outbreak of meningitis with enterovirus in 65 children that were hospitalized starting with May 2016 until November 2016. We also extend the period of surveillance in the 
first 10 months of the year 2017. Rapid diagnosis was performed mainly using the sings and symptoms first revealed in the pediatric patient, and then we decide admission of the children for lombar puncture.

\section{Material and method}

The analyzed group was selected after fulfilling a set of data such as: demographic affiliation, therapeutically standards, laboratory and clinical findings. In order to publish data parents/caregivers inform consent was obtained and also approval of the ethics committee of the Clinical Infectious Diseases Hospital of Constanta.

Suspected cases of viral meningitis were identified based on the following criteria: fever with axillary measurement $>38^{\circ} \mathrm{C}$, vomiting at least 1 episode in $24 \mathrm{~h}$, headache, photophobia, meningeal irritation signs (Kernig or Brudzinski signs, or neck stiffness), impaired consciousness. For each suspected case, demographic data, predominant clinical signs and symptoms, prior history of use of antimicrobial agent, and laboratory results were recorded by using a standardized case report form.

Cerebrospinal fluid (CSF) samples were obtained from all patients with clinical suspected meningitis. CSF samples that had more than 10 leukocytes/mmc and positive Pandy reaction were included in further analysis. No neonates or children under the age of 1 were included in the study.

\section{Results}

Over the studied period (May 2016 - October 2017), 243 patients were admitted with suspicion of Meningitis, but just 73 of them were confirmed (30.04\%). During the mentioned period, a total of
73 patiens, aged 1 to 12 years, were hospitalized with a clinical diagnosis of meningoencephalitis and a cerebrospinal fluid sample was obtained from all of them. All of the patiens were diagnosed at their first episode of meningitis.

Evolution of cases with viral meningitis over the studied period is presented in Figure 1. In year 2016 there were noticed 65 cases while in year 2017 there were registered just 8 cases. In our cohort of children the highest number of cases were registered in September and October 2016 with 23 cases in each month. In August 2016 were registered just 6 cases and in November 2016 -10 cases. First two cases were registered in May 2016.

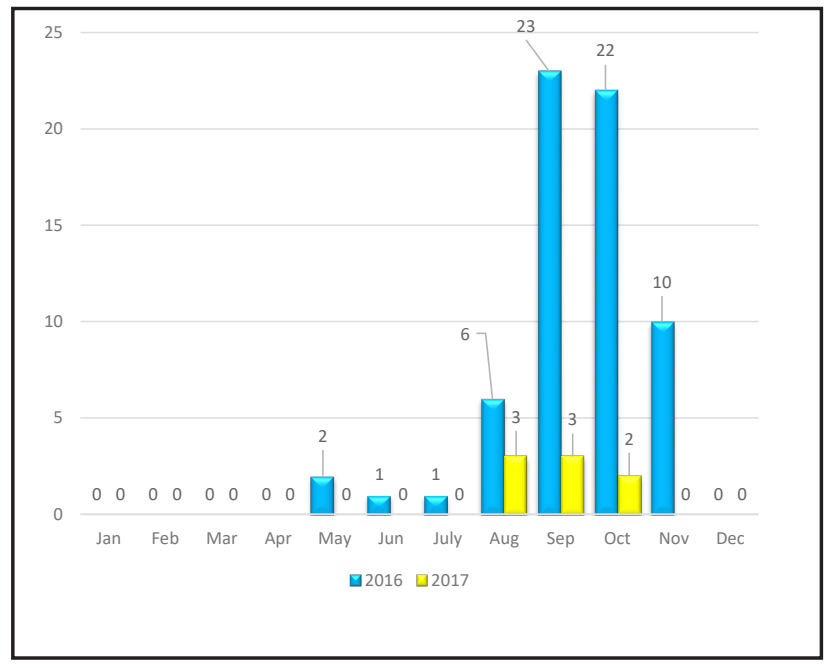

Figure 1. Evolution of Enterovirus Meningitis: May 2016 - October 2017

The average age of the lot was 5 years and 8 months old, with a gender distribution in the favor of boys by a ratio of $2: 1$.

Enviromental area distribution (Figure 2) evidenced a majority of cases from the urban areas with a total of 54 cases out of the 73 cases studied. In comparison, only 19 cases were from rural areas. 


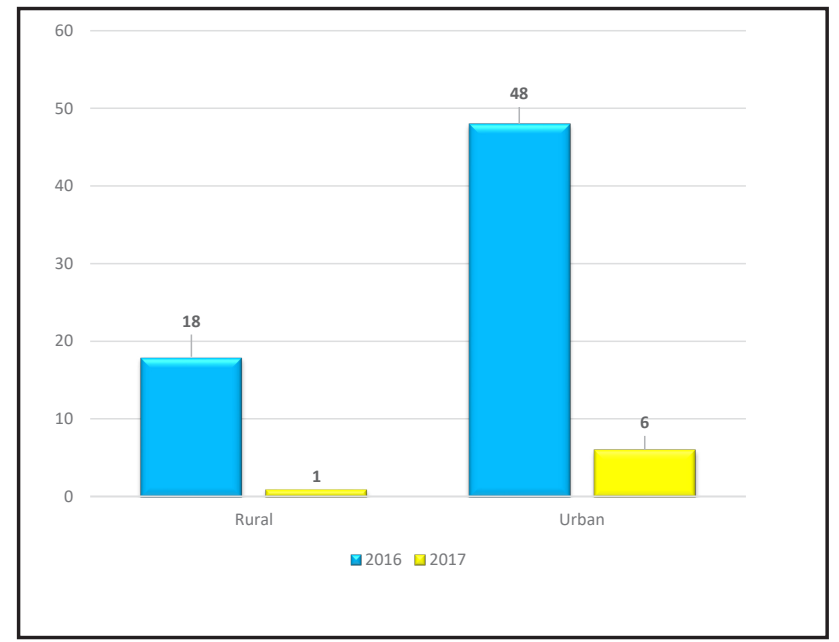

Figure 2 Repartition of Enterovirus Meningitis by environmental area

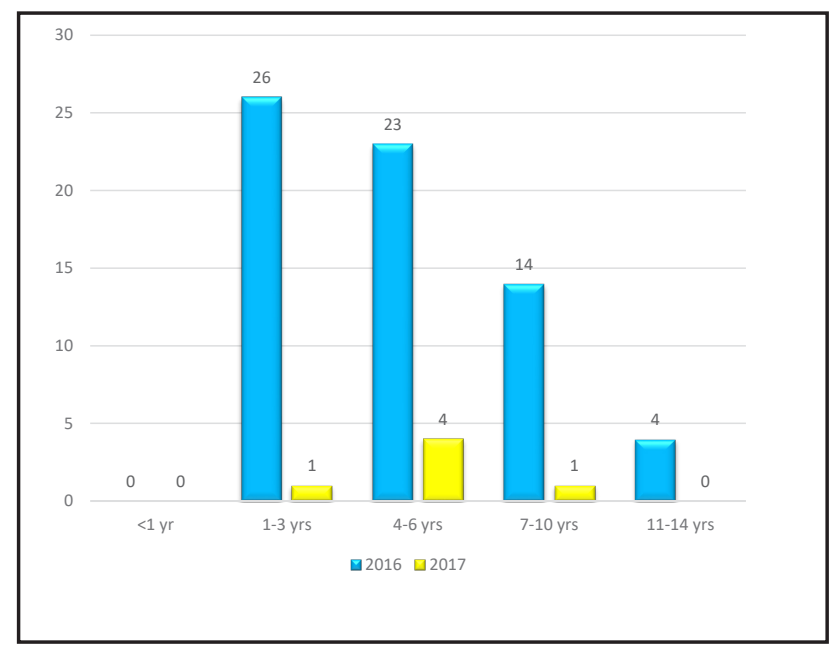

Figure 3 Repartition of Enterovirus meningitis by groups of age

Analysis by age reveals the most affected group of age were 1-3 years and 4-6 years with 27 children each (Figure 3). Age was not a decisive factor in the monthly distribution of the cases, nor in the symptomatic manifestation of the disease.

The study revealed in 53 cases symptoms such as headache, fever and vomiting combined as a determining factor in the decision making for lombar puncture.

Headache was the most common symptom accused by 66 patients, 63 patients were admited for fever with axillary temperature measurement over $38^{\circ} \mathrm{C}$, and 59 children presented vomiting with at least 1 episode in $24 \mathrm{~h}$, usually 3 or more (Figure 4).

Photophobia was presented by 22 of the pediatric patients, all of them associating headache as the main symptom.

Severe symptoms such as obnubilation were present in 5 cases, 4 boys and 1 girl ages 4 to 6 years.

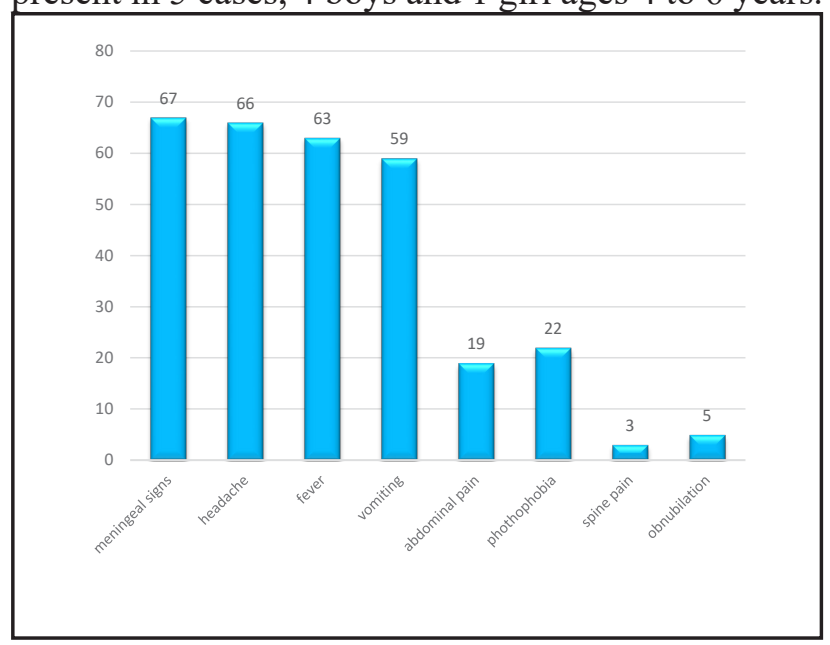

Figure 4 Symptoms and signs in patients with Enterovirus Meningitis

Meningeal irritation signs (at least two) were found in 63 of the studied cases, and only Kernig's sign was found in 4 patients ( 3 girls and 1 boy, ages 4 to 9 years old).

Polymerase chain reaction (PCR) on cerebrospinal fluid (CSF) revealed Enterovirus in 27 out of the 73 cases, 11 girls and 16 boys (Figure 5).

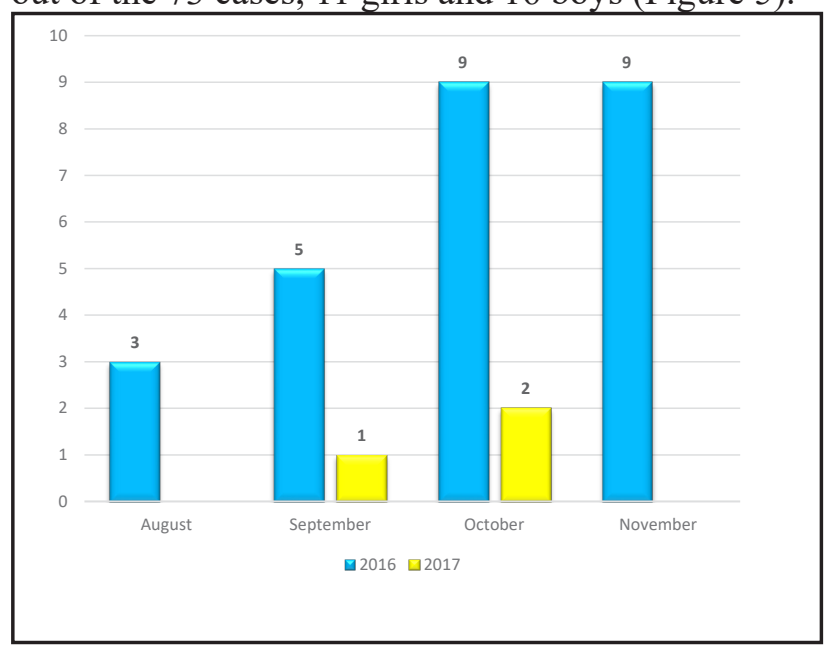

Figure 5 Enterovirus identification in CSF by PCR 
All of the episodes were community-acquired. A positive epidemiological history was recorded for 24 patients - children from the same kindergarten. For other 4 patients an epidemiological link was established through family contact, exactly 2 couples, brother and sister.

We noticed positive serology for Coxsakie viruses in 5 cases in year 2016 while Echovirus was noticed in one case in year 2017.

CSF white cell counts were between 10-1200 cells $/ \mathrm{ml}$, mostly lymphocytes (65/73 cases), as we can see in Figure 5. In year 2016 CSF was clear in 58 cases, meanwhile in 15 cases the fluid was opalescent. CSF analysis revealed postive Pandy reaction in 18 cases, normal glucose levels for every sample and higher proteins levels in only 14 cases.

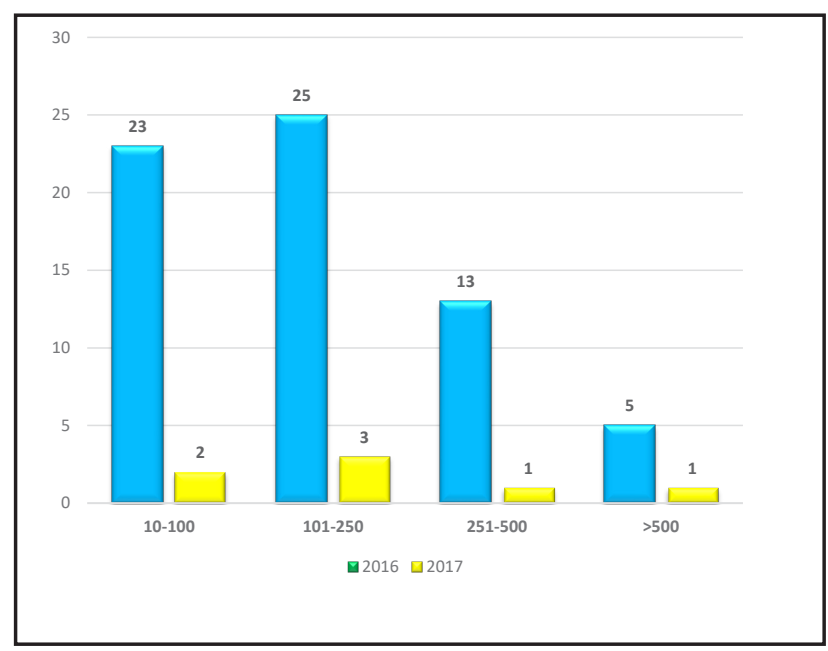

Figure 6 Repartition of cases with enterovirus meningitis by cells/mmc in CSF

Increased white cell count was shown in 50 cases but inflammatory immune biomarkers had no precise specificity.

Anemic syndrome frequency was low, with only 10 cases, the most affected age group was 3-5 years old, especially in boys, with a sex distribution of 9 boys to 1 girl.

Pathogenic and symptomatic treatment was performed in all cases. At this moment, there is no etiologic treatment available for enteroviruses in Constanta. There was no in-hospital mortality in our study.

\section{Discussions}

Viral meningitis is a world known disease, an important source of morbidity and mortality, and an important cause of admission to hospital. Many different viruses can cause meningitis.

The most common viral meningitis are those involving enteroviruses. [1,2] Detection of virus in CSF by PCR was the standard diagnostic tool for meningoencephalitis with enterovirus in our study.

Although in study performed by Graham and Murdoch in enterovirus meningitis, they found a polymorphonuclear cell response in CSF in over a half of the patients in our study proportion of polymorphonuclear cells in CSF was observed just in 8/73 children. [4] Although viral meningitis has been most commonly observed in school-aged children, meningitis with enteroviruses is often observed in preschool children, as can be seen in our case study. [2] In our study the most affected age group were 1-3 years and 4-6 years, with 27 children each.

The most frequent symptoms included sudden fever, headache and vomiting, as shown in medical literature, with 53 cases, in our study, that include all 3 symptoms associated.

The only one investigations useful to establish diagnosis of viral meningitis require cerebrospinal fluid sample by lumbar puncture.

Enteroviruses generally are responsible for mild and moderate diseases. In rare situation, these viruses can cause meningitis or myocarditis with severe evolution [2]. In a study performed by Graaf et al in children hospitalized in South England, they noticed that echoviruses were found in the meningitis group, and Coxsackie B viruses were involved in the myocarditis group [6].

In study performed by Dagan $\mathrm{R}$ et al they found that Echovirus 30 and 11 and Coxsakie B were the most frequent involved in enterovirus meningitis in children [7]. In our studied period children with meningitis presented blood serology positive for Coxsakie virus in 5 cases and for Echovirus in one case.

At this time in meningitis with enteroviruses, 
there is no etiologic treatment although there are some studies that evidenced good results with two antiviral agents: pleconaril or rupintrivir $[8,9]$.

\section{Conclusions}

In our area viral meningitis with enteroviruses should be considered in any paediatric patients admitted with fever, headaches and vomiting, boys and girls, due to the potential outbreaks that can appear during the summer or autumn.

The positive diagnosis of CSF by PCR for enteroviruses is clinically useful for optimizing therapy and disease management.

\section{References}

1. Kaplan, S.L. \& Di Pentima, C., (2017) Patient education: Meningitis in Children (Beyond the Basics). Up To Date, Accessed November 6, 2017. https://www.uptodate.com/contents/ meningitis-in-children-beyond-the-basics

2. Chadwick, D.R. (2005). Viral meningitis. British medical bulletin. 75(1), 1-14.

3. Ihekwaba, U.K., Kudesia, G. \& McKendrick, M.W. (2008). Clinical features of viral meningitis in adults: significant differences in cerebrospinal fluid findings among herpes simplex virus, varicella zoster virus, and enterovirus infections. Clinical infectious diseases. 47(6), 783-789 https://doi.org/10.1086/591129

4. Graham, A.K. \& Murdoch, D.R. (2005). Association between cerebrospinal fluid pleocytosis and enteroviral meningitis. Journal of clinical microbiology. 43(3), 1491-1491. doi: $10.1128 / \mathrm{JCM} .43 .3 .1491 .2005$

5. Henquell, C., Chambon, M., Bailly, J.-L., Alcaraz,
S., De Champs, C., Archimbaud, C., Labbé, A., Charbonné, F. \& Peigue-Lafeuille, H. (2001). Prospective analysis of 61 cases of enteroviral meningitis: interest of systematic genome detection in cerebrospinal fluid irrespective of cytologic examination results. Journal of clinical virology. 21(1), 29-35.

6. de Graaf, H., Pelosi, E., Cooper, A., Pappachan, J., Sykes, K., MacIntosh, I., Gbesemete, D., Clark, T.W., Patel, S.V. \& Faust, S.N. (2016). Severe enterovirus infections in hospitalized children in the South of England: clinical phenotypes and causative genotypes. The Pediatric infectious disease journal. 35(7), 723-727;

7. Dagan, R., Jenista, J.A. \& Menegus, M.A. (1988). Association of clinical presentation, laboratory findings, and virus serotypes with the presence of meningitis in hospitalized infants with enterovirus infection. The Journal of pediatrics. 113(6), 975-978.

8. Desmond, R., Accortt, N., Talley, L., Villano, S., Soong, S.-J. \& Whitley, R. (2006). Enteroviral meningitis: natural history and outcome of pleconaril therapy. Antimicrobial agents and chemotherapy. 50(7), 2409-2414.

9. Zhang, X.-N., Song, Z.-G., Jiang, T., Shi, B.-S., Hu, Y.-W. \& Yuan, Z.-H. (2010). Rupintrivir is a promising candidate for treating severe cases of Enterovirus-71 infection. World journal of gastroenterology: WJG. 16(2), 201-209 doi: 10.3748/wjg.v16.i2.201. 